\title{
Bipolar transurethral vaporization: a superior procedure in benign prostatic hyperplasia: a prospective randomized comparison with bipolar TURP
}

Siavash Falahatkar, Gholamreza Mokhtari, Keivan Gholamjani Moghaddam, Ahmad Asadollahzade, Alireza Farzan, Elaheh Shahab, Ali Ghasemi, Aliakbar Allahkhah, Samaneh Esmaeili

Urology Research Center, Razi Hospital, Guilan University of Medical Sciences

\section{ABSTRACT}

Objective: To compare the outcomes of bipolar transurethral vaporization of the prostate (TUVP) with bipolar transurethral resection of the prostate (TURP).

Materials and Methods: In a prospective randomized trial, 88 patients with moderate to severe lower urinary tract symptoms secondary to benign prostatic hyperplasia (BPH) underwent bipolar TUVP $(\mathrm{N}=39)$ or bipolar TURP $(\mathrm{N}=49)$ from October 2010 to November 2011. The inclusion criteria were age $>50$ years, prostate volume of $30-80 \mathrm{~mL}$, serum PSA $<4 \mathrm{ng} / \mathrm{mL}$, IPSS $\geq 20, \mathrm{Q}_{\max } \leq 10 \mathrm{~mL} / \mathrm{s}$ and failed medical therapy. The perioperative and postoperative outcomes were evaluated and the IPSS and $Q_{\max }$ were assessed preoperatively and 3 months after procedure in all cases.

Results: Both groups were similar in patient age, prostate volume, preoperative IPSS and $\mathrm{Q}_{\max }$. The TUVP group had significantly lower mean values of operative time, hospital stay, catheterization period, irrigation fluid volume and serum hemoglobin, creatinine, sodium and potassium changes compared with TURP group. No significant differences were seen between two groups regarding complications (TUVP $=10.3 \%$; TURP $=12.2 \%$ ) and modified Clavien classification of complications. No TUR syndrome, obturator reflex or epididymitis occurred in both groups. Re-hospitalization and transfusion due to clot retention $(\mathrm{N}=2)$ and urethral stricture $(\mathrm{N}=1)$ were reported only in the TURP group. Three patients experienced urinary retention after catheter removal in the TUVP group. Two patients were re-catheterized temporarily and one patient required repeat bipolar TUVP. Three months after surgery, two groups had significant improvement in IPSS and $\mathrm{Q}_{\max }$. But the TUVP group had significantly lower IPSS and higher $\mathrm{Q}_{\max }$ than TURP group. Conclusions: Bipolar TUVP is a safe, effective and low cost procedure among minimally invasive surgeries of BPH. Compared with bipolar TURP, the bipolar TUVP had similar complications, better perioperative and postoperative outcomes, superior hemostasis and higher efficacy.

\section{ARTICLE INFO}

\section{Key words:}

Transurethral Resection of

Prostate; Prostatic Hyperplasia;

Lower Urinary Tract Symptoms

Int Braz J Urol. 2014; 40: 346-55

Submitted for publication:

August 03, 2013

Accepted after revision:

November 04, 2013

\section{INTRODUCTION}

Benign prostatic enlargement is a common problem in the aging men (1-3) and may lead to lower urinary tract symptoms (LUTS) (2-6).
Several endourologic minimally invasive procedures have been suggested and used for surgical treatment of moderate to severe LUTS related to benign prostatic hyperplasia (BPH) (3,6-11). Although conventional monopolar transurethral 
resection (TUR) of the prostate (TURP) has been applied as a gold standard surgical modality in the recent decades (6-12), but its outcomes including patient discomfort, prolonged catheterization and hospitalization durations, need to transfusion and re-admission due to hemorrhage or clot retention, high volume of irrigation fluid, electrolyte imbalance and TUR syndrome, urinary retention, urethral stricture, incontinence, retrograde ejaculation or erectile dysfunction (6-8,13-20) elicited the tendency to the use of the newer technologies such as bipolar energy or laser energy for resection, vaporization, ablation or enucleation and changing the irrigation fluid from glycine to normal saline in the endoscopic management of $\mathrm{BPH}$ (6,8,13-15,20-26).

Bipolar technology using saline conductive medium accompanies with proper translucency, similar osmolality to the serum, minimal risk of dilutional hyponatremia and TUR syndrome, larger removal of prostate tissue, smaller coagulation depth, longer time for safe resection and coagulation, less tissue damage due to lower energy, and lower temperature and thermal damage $(7,8,12,15,19,27,28)$. Nowadays, bipolar TURP is used in many centers due to several benefits and superior or similar results in comparison with monopolar TURP $(8,14,23,29-31)$. Bipolar transurethral vaporization of the prostate (TUVP) in saline using hovering technique is an easy learning and low cost procedure in comparison to laser technique $(12,14,20)$. Bipolar TUVP provides suitable depth of coagulation, high hemostasis and proper outcomes in comparison with monopolar TUVP and monopolar TURP $(7,14,20,24,26,32-34)$.

In this study, we compared the perioperative and postoperative outcomes of bipolar TUVP and bipolar TURP in a prospective randomized trial in patients with moderate to severe LUTS secondary to $\mathrm{BPH}$.

\section{MATERIALS AND METHODS}

From October 2010 to November 2011, 88 patients underwent bipolar TUVP (B-TUVP, $\mathrm{N}=$ 39) or bipolar TURP (B-TURP, $N=49$ ) in a single-center prospective randomized trial who were referred for surgical management of moderate to se- vere LUTS secondary to BPH. All procedures were performed successfully by single surgeon under spinal anesthesia. Local ethical committee approved this research. Before operation, all patients were assessed by medical history, general physical and regional neurological examinations, digital rectal examination (DRE), laboratory tests (CBC, hemoglobin and hematocrit, coagulation tests, serum prostate specific antigen [PSA], ESR, BUN, serum creatinine and electrolytes [sodium, potassium], urine analysis, urine culture and sensitivity test), abdominal ultrasonography (including measurement of the prostate volume), international prostate symptom score (IPSS) questionnaire and uroflowmetry (measurement of maximum flow rate $\left[Q_{\max }\right]$ ).

The inclusion criteria were age $>50$ years, prostate volume of $30-80 \mathrm{~mL}$, serum PSA $<4 \mathrm{ng} /$ $\mathrm{mL}$, IPSS $\geq 20, \mathrm{Q}_{\max } \leq 10 \mathrm{~mL} / \mathrm{s}$ and failed BPH-related medical therapy. The exclusion criteria were abnormal DRE or ultrasonography with suspicion of prostate cancer, history of prostate cancer, serum PSA $\geq 4 \mathrm{ng} / \mathrm{mL}$, previous urethral or prostate surgery, urethral stricture, neurogenic bladder, bladder calculi, BPH-related hydronephrosis, anticoagulant therapy, coagulation disorders, renal insufficiency and severe co-morbidities or co-existing diseases.

The procedures were accomplished under direct sight by continuous flow irrigation with normal saline $(0.9 \% \mathrm{NaCl})$, the Olympus bipolar generator (cutting: $280 \mathrm{~W}$; coagulation: $125 \mathrm{~W}$ ), the classical and traditional surgical steps of transurethral resection in saline (TURis) for TURP and hovering technique for TUVP. A standard resection loop and a "button-type" vaporization electrode were used for TURis and vaporization without resection respectively. In all patients, a $20-24 \mathrm{Fr}$ three-way Foley catheter was placed at the end of the procedure.

The parameters including operative time, irrigation fluid volume, catheterization period, postoperative hospital stay, complications and postoperative changes in hemoglobin, serum creatinine, sodium and potassium levels were assessed in all patients. Also three months after procedure, the IPSS and $Q_{\max }$ were measured for evaluation of efficacy in all cases. 
Software SPSS version 16.0, independent t test, Mann-Whitney test, Chi-square test, Fisher Exact test and Wilcoxon test were applied for statistical analysis and $\mathrm{P}$-value $<0.05$ was considered significant.

\section{RESULTS}

The mean patients' age, values of prostate volume, IPSS and $\mathrm{Q}_{\max }$ are mentioned in Table-1. The mean patients' age $(70.97 \pm 3.79$ vs. 69.14 \pm 4.09 years) and mean prostate volume (46.92 \pm 4.67 vs. $47.14 \pm 4.44 \mathrm{~mL}$ ) were similar between B-TUVP and B-TURP groups. No significant differences were seen in pre-operative IPSS $(\mathrm{P}=0.545)$ and $\mathrm{Q}_{\max }(\mathrm{P}=0.341)$ between B-TUVP $(26.36 \pm$
$1.96 ; 8.48 \pm 1.04 \mathrm{~mL} / \mathrm{s})$ and B-TURP $(26.04 \pm 3.02$; $8.22 \pm 1.21 \mathrm{~mL} / \mathrm{s}$ ) groups. (Table-2).The mean values of operative time $(25.92 \pm 2.36$ vs. $32.63 \pm$ 2.87 minutes), postoperative hospital stay (1.89 \pm 0.38 vs. $2.10 \pm 0.51$ days) (hospitalization was calculated by the time the patients were discharged after the operation), catheterization period (4.12 \pm 0.33 vs. $4.77 \pm 0.42$ days) and irrigation fluid volume $(10.74 \pm 1.46$ vs. $14.22 \pm 1.04$ liters $)$ in the B-TUVP group were significantly $(\mathrm{P}<0.05)$ lower than the B-TURP group. Compared with the B-TURP group, the mean hemoglobin drop $(0.53 \pm$ 0.29 vs. $1.39 \pm 0.45 \mathrm{~g} / \mathrm{dL}$ ) and the mean changes in serum creatinine, sodium and potassium levels were significantly $(\mathrm{P}<0.05)$ lower for the B-TUVP group (Table-1).

Table 1 - Patient- and operation-related parameters in Bipolar TUVP and Bipolar TURP groups.

\begin{tabular}{lccc}
\hline Parameter & Bipolar TUVP & Bipolar TURP & P-value \\
\hline Patients, $\mathrm{n}$ & 39 & 49 & - \\
Mean Age (range; SE), year & $70.97 \pm 3.79$ & $69.14 \pm 4.09$ & $0.053^{*}$ \\
& $(65-79 ; 0.60)$ & $(62-77 ; 0.58)$ & \\
Mean Prostate Volume (SE), mL & $46.92 \pm 4.67$ & $47.14 \pm 4.44$ & $0.888^{*}$ \\
& $(0.74)$ & $(0.63)$ & \\
Mean Operative Time (range; SE), minute & $25.92 \pm 2.36$ & $32.63 \pm 2.87$ & $<0.001^{*}$ \\
& $(22-35 ; 0.37)$ & $(25-35 ; 0.41)$ & \\
Mean Irrigation Fluid Volume (range; SE), liter & $10.74 \pm 1.46$ & $14.22 \pm 1.04$ & $<0.001^{*}$ \\
& $(8-13 ; 0.23)$ & $(12-15 ; 0.15)$ & \\
Mean Postoperative Hospital stay (range; SE), day & $1.89 \pm 0.38$ & $2.10 \pm 0.51$ & $0.047^{\star}$ \\
Mean Postoperative Catheterization Period (range; SE), day & $(1-3 ; 0.06)$ & $(1-4 ; 0.07)$ & \\
& $4.12 \pm 0.33$ & $4.77 \pm 0.42$ & $<0.001^{*}$ \\
Mean Hemoglobin Drop (range; SE), g/dL & $(4-5 ; 0.05)$ & $(4-5 ; 0.06)$ & \\
& $0.53 \pm 0.29$ & $1.39 \pm 0.45$ & $<0.001^{\S}$ \\
Mean Serum Creatinine Level Increase (range; SE), mg/dL & $(0-1.4 ; 0.04)$ & $(0.5-3 ; 0.06)$ & \\
Mean Serum Sodium (Na+) Level Decrease (range; SE), & $0.10 \pm 0.08$ & $0.16 \pm 0.33$ & $0.338^{*}$ \\
meq/l & $(0-0.3 ; 0.01)$ & $(0-2 ; 0.04)$ & $<0.001^{\S}$ \\
Mean Serum Potassium (K+) Level Increase (range; SE), & $1.71 \pm 0.19$ & $2.29 \pm 0.15$ & $0.012^{\S}$ \\
meq/l & $(1.3-2.2 ; 0.03)$ & $(2-2.6 ; 0.02)$ & $0.31 \pm 0.13$ \\
\hline
\end{tabular}

$\mathbf{S E}=$ Standard error of mean; ${ }^{*}=$ Mann-Whitney test; $\boldsymbol{\S}=$ Independent $\mathrm{T}$ test 
Table 2 - IPSS and Qmax in Bipolar TUVP (B-TUVP) and Bipolar TURP (B-TURP) groups.

\begin{tabular}{lccc}
\hline & Before Operation & After Operation & P-value \\
\hline B-TUVP: mean IPSS (range; SE) & $26.36 \pm 1.96(20-30 ; 0.31)$ & $2.56 \pm 2.58(0-10 ; 0.41)$ & $<0.001^{*}$ \\
B-TURP: mean IPSS (range; SE) & $26.04 \pm 3.02(20-30 ; 0.43)$ & $5.49 \pm 3.40(0-10 ; 0.48)$ & $<0.001^{*}$ \\
P-value & $0.545 \dagger$ & $<0.001 \dagger$ & - \\
B-TUVP: mean Qmax (range; SE), mL/s & $8.48 \pm 1.04(7-10 ; 0.16)$ & $23.23 \pm 1.08(18-24 ; 0.17)$ & $<0.001^{*}$ \\
B-TURP: mean Qmax (range; SE), mL/s & $8.22 \pm 1.21(6-10 ; 0.17)$ & $20.79 \pm 1.47(18-22 ; 0.21)$ & $<0.001^{*}$ \\
P-value & $0.341 \dagger$ & $<0.001 \dagger$ & - \\
\hline
\end{tabular}

SE = Standard error of mean; ${ }^{*}=$ Wilcoxon test; $\boldsymbol{\dagger}=$ Mann-Whitney test

The overall complication rate was $10.3 \%$ in the B-TUVP group and $12.2 \%$ in the B-TURP group. No significant differences $(\mathrm{P}>0.05)$ were seen in overall complications and modified Clavien classification of complications between the B-TUVP and the B-TURP groups. Low grade self-limited fever occurred in one patient $(2.6 \%)$ of the B-TUVP group and in three patients $(6.1 \%)$ of the B-TURP group and this difference was non-significant $(\mathrm{P}=0.626)$. No TUR syndrome, obturator reflex or epididymitis were seen in both groups. Two patients (4.1\%) experienced postoperative hematuria and clot retention during one week after B-TURP procedure and were re-hospitalized. These patients were treated by bladder fluid irrigation and required blood transfusion. Urinary retention occurred after catheter removal in the three patients who underwent B-TUVP. Two patients were re-catheterized for a short period and one patient re-treated with repeat B-TUVP. During a three month follow-up, urethral stricture occurred only in one patient following B-TURP who was treated by endoscopic urethral dilation (Table-3).

Three months after procedure, significant $(\mathrm{P}<0.001)$ improvement in the mean values of IPSS and $Q_{\max }$ were seen in the B-TUVP and the B-TURP groups. But the B-TUVP had significantly higher efficacy compared with B-TURP. The B-TUVP group $(2.56 \pm 2.58)$ had significantly $(\mathrm{P}<0.001)$ lower mean IPSS than the B-TURP group $(5.49 \pm 3.40)$. Also in the B-TUVP group $(23.23 \pm 1.08 \mathrm{~mL} / \mathrm{s})$, mean $\mathrm{Q}_{\max }$ was significantly
$(\mathrm{P}<0.001)$ higher than B-TURP group $(20.79 \pm$ $1.47 \mathrm{~mL} / \mathrm{s}$ ) (Table-2).

\section{DISCUSSION}

The quality of life and health care cost may be affected in the aging males due to LUTS following benign progressive enlargement of the prostate gland $(1-6,31,35)$. The monopolar TURP has been used as the traditional therapeutic procedure of LUTS/BPH for several decades (6-12,36,37). But for reducing adverse events such as bleeding and clot retention, TUR syndrome, damage of surrounding or deeper tissues, and urethral stricture, bipolar technology and conductive irrigation fluid of normal saline have been applied instead of monopolar energy and glycine nonconductive medium as the popular and the most important alternatives in the recent years $(18,23,28,29,31,36)$. The bipolar TURP is a proper procedure with shorter learning curve (30), better outcomes concerning duration of irrigation and catheterization (31), depths of coagulation zone (28), decrease in serum sodium $(15,29,31,38)$, occurrence of TUR syndrome $(18,29,31,39)$ bleeding $(23,28,38)$, clot retention $(31,39)$ and re-admission (23), comparative efficacy and other similar results in comparison with M-TURP $(15,23,29-31,38)$.

Increase in life expectancy and higher prevalence of surgical risk and comorbid diseases such as cardio-pulmonary diseases, coagulation disorders and anti-platelet or anticoagulant therapies in the 
Table 3 - Complications in Bipolar TUVP and Bipolar TURP groups.

\begin{tabular}{|c|c|c|c|}
\hline Parameter & Bipolar TUVP & Bipolar TURP & P-value \\
\hline Complication, n (\%) & $4(10.3 \%)$ & $6(12.2 \%)$ & $1.000^{*}$ \\
\hline TUR syndrome, $\mathrm{n}(\%)$ & $0(0 \%)$ & $0(0 \%)$ & - \\
\hline Obturator Reflex, n (\%) & $0(0 \%)$ & $0(0 \%)$ & - \\
\hline Epididymitis, n (\%) & $0(0 \%)$ & $0(0 \%)$ & - \\
\hline Fever, $\mathrm{n}(\%)$ & $1(2.6 \%)$ & $3(6.1 \%)$ & $0.626^{*}$ \\
\hline Postoperative Hematuria, n (\%) & $0(0 \%)$ & $2(4.1 \%)$ & $0.501^{*}$ \\
\hline Transfusion, $\mathrm{n}(\%)$ & $0(0 \%)$ & $2(4.1 \%)$ & $0.501^{*}$ \\
\hline Clot Retention, n (\%) & $0(0 \%)$ & $2(4.1 \%)$ & $0.501^{*}$ \\
\hline Re-hospitalization, n (\%) & $1(2.6 \%)$ & $2(4.1 \%)$ & $1.000^{*}$ \\
\hline Urinary Retention, $\mathrm{n}(\%)$ & $3(7.7 \%)$ & $0(0 \%)$ & $0.083^{*}$ \\
\hline Re-catheterization, n (\%) & $2(5.1 \%)$ & $0(0 \%)$ & $0.194^{*}$ \\
\hline Repeat Surgery, n (\%) & $1(2.6 \%)$ & $0(0 \%)$ & $0.443^{*}$ \\
\hline Urethral Stricture, n (\%) & $0(0 \%)$ & $1(2.0 \%)$ & $1.000^{*}$ \\
\hline \multicolumn{4}{|c|}{ Modified Clavien Classification of Complications } \\
\hline Grade $0, \mathrm{n}(\%)$ & $35(89.7 \%)$ & $43(87.8 \%)$ & $0.707 \dagger$ \\
\hline Grade I, n (\%) & $1(2.6 \%)$ & $3(6.1 \%)$ & \\
\hline Grade II, n (\%) & $0(0 \%)$ & $0(0 \%)$ & \\
\hline Grade III, n (\%) & $3(7.7 \%)$ & $3(6.1 \%)$ & \\
\hline Grade IV, $\mathrm{n}(\%)$ & $0(0 \%)$ & $0(0 \%)$ & \\
\hline Grade V, n (\%) & $0(0 \%)$ & $0(0 \%)$ & \\
\hline
\end{tabular}

${ }^{*}=$ Fisher Exact test; $\boldsymbol{\dagger}=$ Chi-Square test

old patients may lead to the limitation in the use of bipolar TURP $(3,18)$. Also fluid absorption betides in the bipolar TURP and volume overload may be problematic in patients with severe cardio-pulmonic disorders $(23,27,31,36)$. In the last decade, laser energy has been applied with high safety and efficacy for vaporization or enucleation of hypertrophic large volume prostate $(6,9,11-13,40-42)$. But laser therapy may be accompanied with the use of multiple techniques and different wattage, technical complexity, prolonged operative time, higher applied energy and re-operation rate in the larger prostate volume cases, high cost equipment and inaccessibility in many centers $(6,9,11-13,40-$ 42). Beside morbidity and complication, attention to other outcomes, prostate volume, anesthesia risk, patient satisfaction, cost-benefit or cost-effectiveness, learning curve, easy accessing and performing is important in the selection of preferable procedure.

The bipolar TUVP (including the plasmakinetic vaporization of the prostate [PKVP] using the Gyrus system and the technique of TURis bipolar plasma vaporization using Olympus generator) profits by advantages of monopolar TURP and bipolar TURP and is devoid of their 
limitations in the high risk patients $(9,12,34,43)$. Also the bipolar TUVP can compete with TURP and laser-related techniques due to easy learning and lower cost procedure, superior hemostasis, decreased fluid absorption and TUR syndrome, low morbidity and comparative outcomes $(9,12,14,34)$. In a prospective randomized trial, we compared the outcomes between bipolar TUVP and bipolar TURP in patients with moderate to severe LUTS/ $\mathrm{BPH}$. The mean values of pre- and postoperative hemoglobin were 13.8-14.3 and 12.7-13.1 $\mathrm{g} / \mathrm{dL}$ without transfusion requirement in two experiences about bipolar TUVP (B-TUVP) $(12,20)$. Dunsmuir and co-workers reported similar postoperative hemoglobin between bipolar electrovaporization and TURP (16). But in Hon et al. study, bipolar PKVP $(0.8 \mathrm{~g} / \mathrm{dL})$ had significantly lower hemoglobin drop compared with standard TURP (1.39g/dL) (17). The mean hemoglobin drop in the B-TUVP $(0.5 \mathrm{~g} / \mathrm{dL})$ was significantly $(\mathrm{P}=0.0001)$ lower than bipolar TURP (B-TURP) $(1.2 \mathrm{~g} / \mathrm{dL})$ and monopolar TURP (M-TURP) $(1.6 \mathrm{~g} / \mathrm{dL})$ in Geavlete et al. randomized comparison (34). Also in our trial, the B-TUVP had lesser mean hemoglobin drop than B-TURP (0.53 vs. $1.39 \mathrm{~g} / \mathrm{dL}$ ) due to superior hemostasis and coagulation $(12,34)$.

The published experiences reported the mean postoperative hospitalization and catheterization periods of 1.4-2.08 and 2.2-3.54 days for bipolar vaporization $(12,20,44,45)$. No significant differences were seen in catheterization and hospitalization duration between bipolar electrovaporization (1193 minutes, 1.45 days) and TURP (1007 minutes, 1.5 days) in Dunsmuir and co-workers trial (16). But catheterization time was significantly shorter for vaporization compared with TURP in multiple studies (1.9 vs. 2.71 days (46). PKVP: 35 vs. 68 hours (47), bipolar plasmakinetic vaporization-resection: 2.3 vs. 3.8 days (19), B-TUVP: 1.3 vs. 2.8 days (14). Also vaporization had significantly shorter hospital stay than TURP (3.9 vs. 4.7 days (46), PKVP: 3.02 vs. 3.36 days (17)). The photoselective vaporization of the prostate (PVP) using laser energy and B-TURP had significantly shorter catheterization and hospitalization times compared with M-TURP in the different meta-analysis studies $(6,22,31)$. In Geavlete et al. randomized comparison, the mean catheterization and hospitalization times were 23.5 hours and 1.9 days in the B-TUVP (the shortest), 46.3 hours and 3.1 days in the B-TURP, 72.8 hours and 4.2 days in the M-TURP (the longest) and these differences were significant $(P=0.0001)$ (34). Proper hemostasis, lower blood loss and hemorrhagic events and subsequent morbidities following B-TUVP can justify shorter postoperative hospitalization (1.89 vs. 2.10 days) and catheterization (4.12 vs. 4.77 days) in comparison with B-TURP in our results (12).

The operating time had the means of 61 and 63 minutes for B-TUVP and the median of 55 minutes for bipolar plasmakinetic electrovaporization in the published experiences $(12,20,45)$. In Dunsmuir and co-workers trial, the bipolar electrovaporization and TURP had similar operation time (33 vs. 26 minutes, $P=0.78$ ) (16). Hon et al. reported longer mean resection time for PKVP compared with standard TURP (32.6 vs. 28.5 minutes, $\mathrm{P}=$ 0.08 ) (17). But in other studies, the PKVP (40.3 vs. 55 minutes) (47) and bipolar plasmakinetic vaporization-resection (40.3 vs. 57.8 minutes, $\mathrm{P}<0.01$ ) (19) had shorter mean operative time than TURP. In the different meta-analysis studies, the operative time was similar between B-TURP and M-TURP (31) but the operative time in the PVP was longer than TURP (6). Ahyai et al. reported the shortest mean operation time (36 minutes) for B-TUVP among minimally invasive surgical therapies (14). Also in Geavlete et al. comparison, the mean operation time in the B-TUVP (39.7 minutes) was significantly ( $\mathrm{P}$ $=0.0001$ ) shorter than B-TURP (52.1 minutes) and M-TURP (55.6 minutes) (34). In our trial, B-TUVP had shorter mean operative time (25.92 vs. 32.63 minutes) compared with B-TURP. This result can be explained by easy performance, good hemostasis and coagulation, better sighting due to decreased hemorrhage, the lack of vacating resected samples and possible proper vaporization of prostatic tissue during moving of the electrode in the B-TUVP $(12,34)$.

The mean values of pre- and postoperative serum sodium were 141.3 and $140.6 \mathrm{mmol} / \mathrm{L}$ and no serum electrolyte abnormality occurred in Reich et al. experience in B-TUVP (12). In Otsuki et al. report the irrigation fluid volume was 22.9 liters and the B-TUVP had similar pre- and postoperative serum sodium level (20). Mamoulakis et al. reported signi- 
ficantly higher sodium level for B-TURP compared with M-TURP (31). In Dunsmuir and co-workers trial, bipolar electrovaporization and TURP had similar postoperative serum sodium level (16). Also similar sodium changes and perioperative fluid absorption were reported for PKVP and TURP in Hon et al. study (17). The bipolar plasmakinetic vaporization-resection had significantly lower mean perioperative irrigation fluid volume compared with TURP (11.4 vs. 18.3 lit) in Tefekli et al. comparison (19). In our study, the mean values of irrigation fluid volume (10.74 vs. 14.22 lit), serum sodium ( 1.71 vs. $2.29 \mathrm{meq} / \mathrm{l})$ and potassium $(0.22$ vs. $0.31 \mathrm{meq} / \mathrm{l})$ changes in the B-TUVP were lower than B-TURP. Reduced hemorrhage, superior sight and performing the operation without resection and sampling, decrease irrigation fluid volume, subsequent fluid absorption and serum electrolytes changes in the B-TUVP compared with B-TURP $(12,34)$.

The early postoperative complication rate in the bipolar plasmakinetic vaporization-resection was higher than TURP (16.3\% vs. 8.5\%, P = 0.0014) in Tefekli et al. (19) research, but the overall complication rate was similar ( $10.2 \%$ vs. $6.3 \%, \mathrm{P}>0.05)$ (19). In the meta-analysis from Ahyai et al., the B-TUVP (14\%) and B-TURP (12\%) had significantly lower perioperative complication rates compared with TURP (18.7\%) (14). But the intraoperative, late and overall complication rates were similar (14). During our follow-up, no significant differences were seen between B-TUVP and B-TURP regarding overall complication rate (10.3\% vs. $12.2 \%)$ and modified Clavien classification of complications.

The complications including gross hematuria requiring re-catheterization and bladder irrigation (4.7\%) (20), transfusion (1.9\%) (20), AUR requiring re-catheterization (5.7\%) (20) transient incontinence (1.9\%) (20), re-catheterization (13\%) (12), bladder neck contracture (0.9\%) (20), urethral stricture $(7.5 \%, 4.7 \%)(20,44)$ and re-operation $(3 \%$, $0 \%)(12,20)$ were reported for bipolar vaporization in the published experiences. In Hammadeh and co-workers study, TUVP and TURP had similar results regarding re-operation, urethral stricture, impotency and retrograde ejaculation (48). In Kaya et al. research, PKVP (12\%) had significantly higher re-operation rate than standard TURP (6.7\%). But urethral stricture, erectile dysfunction and retrograde ejaculation were similar in both groups and no urinary incontinence or bladder neck stricture occurred (43). In Hon et al. (17) and Karaman et al. (47) studies, transfusion occurred only in the TURP group compared with PKVP. The bipolar electrovaporization had significantly higher rate of re-catheterization (30\% vs. 5\%) and lower rate of clot evacuation (0\% vs. 19\%) compared with TURP in Dunsmuir and co-workers trial (16). In Tefekli et al. comparison, the re-operation rate was 4.1\% and $2.1 \%$ in the bipolar vaporization-resection and TURP groups (19). The re-catheterization rate was $4.1 \%$ for vaporization-resection (19). The bipolar vaporization-resection had higher rates of severe irritative symptoms (12.2\% vs. 4.3\%) and urethral stricture (6.1\% vs. $2.1 \%, P=0.002)$ (19). But both groups were similar in terms of transfusion, AUR and re-catheterization, and retrograde ejaculation (19). In the meta-analysis studies, the B-TURP had significantly lower rates of TUR syndrome $(22,31)$ and clot retention $(22,31)$ compared with M-TURP. But both techniques were similar regarding transfusion $(22,31)$ AUR (31), re-operation (22), urethral stricture (31) and bladder neck contracture (31). Although the PVP had significantly lower rates of capsular perforation, TUR syndrome, transfusion and clot retention and higher re-operation rate than TURP in the meta-analysis, both procedures had similar results regarding AUR and urethral/bladder neck sclerosis (6). In the meta-analysis from Ahyai et al., the B-TUVP had higher rate of AUR and re-catheterization (8.2\% vs. 3.6\%, 4.5\%), transient dysuria $(2.9 \%$ vs. $0 \%, 0.8 \%)$ and re-intervention $(2.4 \%$ vs. $0.2 \%, 0.5 \%)$ and lower rates of hematuria $(0.0 \%$ vs. $1.0 \%, 3.5 \%)$ and transfusion $(0.5 \%$ vs. 1.9\%, 2.0\%) compared with B-TURP and TURP (14). The urgency (2.2\% vs. $0 \%, 0.2 \%)$, bladder neck stenosis ( $2 \%$ vs. $0.5 \%, 0.5 \%$ ) and urethral stricture ( $4.1 \%$ vs. $1.9 \%, 2.4 \%)$ in the TURP were higher than B-TUVP and B-TURP (14). Bleeding, capsular perforation and TUR syndrome were reported following TURP and the three techniques had similar results regarding mucosal injury and conversion to TURP (14). In Geavlete et al. randomized comparison, the B-TUVP had significantly lower rates of intraoperative bleeding $(1.8 \%$ vs. $8.2 \%, 13.5 \%)$, capsular perforation (1.2\% vs. 7.1\%, 9.4\%), re- 
-catheterization (1.8\% vs. 5.9\%, 7.1\%), re-treatment (3.5\% vs. 9.4\%, 8.8\%) and bladder neck sclerosis $(0.6 \%$ vs. $3.5 \%, 4.1 \%)$ compared with B-TURP and M-TURP (34). Also postoperative hematuria $(2.9 \%, 4.7 \%$ vs. $15.3 \%)$, transfusion $(1.2 \%, 1.8 \%$ vs. $6.5 \%)$, clot retention (0.6\%, $1.2 \%$ vs. $4.1 \%)$, TUR syndrome ( $0 \%, 0 \%$ vs. $1.8 \%)$ and re-hospitalization following hemorrhage $(0.6 \%, 1.2 \%$ vs. $3.5 \%)$ in the B-TUVP and B-TURP were significantly lower than M-TURP (34). But the three groups had similar results regarding early irritative symptoms, urethral stricture and urinary incontinence (34). During our follow-up, no significant differences were seen between B-TUVP and B-TURP regarding TUR syndrome, re-hospitalization, repeat surgery and urethral stricture. Although postoperative hematuria, clot retention and transfusion occurred only in the B-TURP group and urinary retention and re-catheterization occurred only in the B-TUVP group, these differences were non-significant probably due to the small sample size.

The published studies have reported significant improvement in IPSS and $Q_{\max }$ with different follow-up durations for vaporization and vaporization-resection (including bipolar technology) in the case series $(12,20,44,45)$ or in comparison with monopolar or bipolar TURP $(19,34,43,46-48)$ and our results are similar to those. In the meta-analysis studies, the PVP and B-TURP had similar results with M-TURP regarding IPSS and $Q_{\max }$ especially in the short-term follow-up $(6,14,22,31)$ Otsuki and co-workers reported significant improvement in IPSS between three months and one month after B-TUVP (8.8 vs. 11.1), but this matter wasn't proved about $\mathrm{Q}_{\max }(15.2$ vs. $15.1 \mathrm{~mL} / \mathrm{s})$ (20). Karaman et al. reported significantly higher improvement of IPSS for PKVP compared with TURP on postoperative month 3 and both groups had similar Qmax values (47). In Tefekli et al. comparison, the bipolar plasmakinetic vaporization-resection had significantly higher improvement in $Q_{\max }(120.5 \%$ vs. 103.6\%) compared with TURP at 12 months after operation (19). But both groups had similar improvement regarding IPSS (63\% vs. 64.3\%) (19). But in Kaya et al. comparison, the TURP had significantly better result of IPSS (5.2, 5.7 vs. 7.1, 7.6) and $Q_{\max }(20.8,21.8$ vs. $12.5,14.4 \mathrm{~mL} / \mathrm{s}$ ) than PKVP at 24 and 36 months after operation (43). Furthermore, no significant di- fferences were seen in efficacy (based on IPSS and $\mathrm{Q}_{\max }$ ) between vaporization (including bipolar technology) and TURP in multiple studies $(14,16,17,48)$. In Geavlete et al. trial, the B-TUVP had significantly better results of IPSS and $Q_{\max }$ compared with $B$ -TURP and M-TURP during 18 months of follow-up (34). Also in our trial, the B-TUVP had significantly higher improvement in postoperative IPSS (2.56 vs. 5.49) and $Q_{\max }$ (23.23 vs. $20.79 \mathrm{~mL} / \mathrm{s}$ ) compared with B-TURP. Proper visibility due to lesser bleeding and formation of suitable cavity with good margins and surface in the operated area can explain this result with B-TUVP (34). We believe that TUVP can be a good alternative for TURP or even laser, because of lesser bleeding during the surgery and low cost of the equipments.

\section{CONCLUSIONS}

Bipolar TUVP is a safe, effective and low cost procedure in the endoscopic minimally invasive surgical management of BPH. Compared with bipolar TURP, the bipolar TUVP had similar complications and significantly better perioperative and postoperative outcomes (including shorter operative time and postoperative hospitalization and catheterization periods, lower irrigation fluid volume and serum sodium and potassium changes), superior hemostasis (due to lower hemoglobin drop) and higher efficacy (because of superior improvement in postoperative IPSS and $\mathrm{Q}_{\max }$ ). However, more studies are needed with large amount of patients to corroborate our results.

\section{CONFLICT OF INTEREST}

None declared.

\section{REFERENCES}

1. Verhamme KM, Dieleman JP, Bleumink GS, van der Lei J, Sturkenboom MC, Artibani W, Begaud B, et al.: Incidence and prevalence of lower urinary tract symptoms suggestive of benign prostatic hyperplasia in primary care--the Triumph project. Eur Urol. 2002; 42: 323-8.

2. Wei JT, Calhoun E, Jacobsen SJ: Urologic diseases in America project: benign prostatic hyperplasia. J Urol. 2005; 173: 1256-61. 
3. Strope SA, Yang L, Nepple KG, Andriole GL, Owens PL: Population based comparative effectiveness of transurethral resection of the prostate and laser therapy for benign prostatic hyperplasia. J Urol. 2012; 187: 1341-5.

4. Abrams P, Chapple C, Khoury S, Roehrborn C, de la Rosette $\mathrm{J}$; International Scientific Committee: Evaluation and treatment of lower urinary tract symptoms in older men. $J$ Urol. 2009; 181: 1779-87.

5. Roehrborn C: Benign prostatic hyperplasia and lower urinary tract symptom guidelines. Can Urol Assoc J. 2012; 6(5 Suppl 2): S130-2.

6. Zhang X, Geng J, Zheng J, Peng B, Che J, Liang C: Photoselective vaporization versus transurethral resection of the prostate for benign prostatic hyperplasia: a metaanalysis. J Endourol. 2012; 26: 1109-17.

7. Bucuras V, Bardan R: Bipolar vaporization of the prostate: is it ready for the primetime? Ther Adv Urol. 2011; 3: 25761.

8. Bolgeri M, Naji S, Sahai A, Anjum F, Madaan S, Sriprasad S, Dickinson I: Bipolar transurethral resection of prostate: current status in the management of bladder outflow obstruction. Br J Med Surg Urol 2012; 5: 105-10.

9. Jepsen JV, Bruskewitz RC: Recent developments in the surgical management of benign prostatic hyperplasia. Urology. 1998; 51(4A Suppl): 23-31.

10. Kacker R, Williams SB: Endourologic procedures for benign prostatic hyperplasia: review of indications and outcomes. Urol J. 2011; 8: 171-6.

11. Reich 0 , Gratzke C, Stief CG: Techniques and long-term results of surgical procedures for BPH. Eur Urol. 2006; 49: 970-8; discussion 978.

12. Reich 0 , Schlenker B, Gratzke C, Tilki D, Riecken M, Stief $C$, et al.: Plasma vaporisation of the prostate: initial clinical results. Eur Urol. 2010; 57: 693-7.

13. Lusuardi L, Myatt A, Sieberer M, Jeschke S, Zimmermann R, Janetschek G: Safety and efficacy of Eraser laser enucleation of the prostate: preliminary report. J Urol. 2011; 186: 1967-71.

14. Ahyai SA, Gilling P, Kaplan SA, Kuntz RM, Madersbacher S, Montorsi $F$, et al.: Meta-analysis of functional outcomes and complications following transurethral procedures for lower urinary tract symptoms resulting from benign prostatic enlargement. Eur Urol. 2010; 58: 384-97.

15. Michielsen DP, Debacker T, De Boe V, Van Lersberghe C, Kaufman L, Braeckman JG, et al.: Bipolar transurethral resection in saline--an alternative surgical treatment for bladder outlet obstruction? J Urol. 2007; 178: 2035-9; discussion 2039.

16. Dunsmuir WD, McFarlane JP, Tan A, Dowling C, Downie J, Kourambas J, et al.: Gyrus bipolar electrovaporization vs transurethral resection of the prostate: a randomized prospective single-blind trial with $1 \mathrm{y}$ follow-up. Prostate Cancer Prostatic Dis. 2003; 6: 182-6.
17. Hon NH, Brathwaite D, Hussain Z, Ghiblawi S, Brace H, Hayne D, et al.: A prospective, randomized trial comparing conventional transurethral prostate resection with PlasmaKinetic vaporization of the prostate: physiological changes, early complications and long-term followup. J Urol. 2006; 176: 205-9.

18. Rassweiler J, Teber D, Kuntz R, Hofmann R: Complications of transurethral resection of the prostate (TURP)--incidence, management, and prevention. Eur Urol. 2006; 50: 969-79; discussion 980.

19. Tefekli A, Muslumanoglu AY, Baykal M, Binbay M, Tas A, Altunrende F: A hybrid technique using bipolar energy in transurethral prostate surgery: a prospective, randomized comparison. J Urol. 2005; 174: 1339-43.

20. Otsuki H, Kuwahara Y, Kosaka T, Tsukamoto T, Nakamura K, Shiroki R, et al.: Transurethral resection in saline vaporization: evaluation of clinical efficacy and prostate volume. Urology. 2012; 79: 665-9.

21. Yu X, Elliott SP, Wilt TJ, McBean AM: Practice patterns in benign prostatic hyperplasia surgical therapy: the dramatic increase in minimally invasive technologies. J Urol. 2008; 180: 241-5; discussion 245.

22. Burke N, Whelan JP, Goeree L, Hopkins RB, Campbell K, Goeree R, et al.: Systematic review and meta-analysis of transurethral resection of the prostate versus minimally invasive procedures for the treatment of benign prostatic obstruction. Urology. 2010; 75: 1015-22.

23. Fagerström T, Nyman CR, Hahn RG: Complications and clinical outcome 18 months after bipolar and monopolar transurethral resection of the prostate. J Endourol. 2011; 25: 1043-9.

24. Qu L, Wang $X$, Huang $X$, Zhang $Y$, Zeng $X$ : Use of a novel ex-vivo model to compare the hemostatic properties of plasmakinetic resection, transurethral vaporization resection and conventional transurethral resection of the prostate. Urology. 2007; 70: 1034-8.

25. Ponholzer A, Marszalek M, Madersbacher S. Minimally invasive treatment of $\mathrm{BPH}$ : an update. EAU update series 2004; 2:24-33.

26. Illing R: Surgical and minimally invasive interventions for LUTS/BPH: highlights from 2006. European Urology Supplements 2007; 6: 701-9.

27. Reich 0: Bipolar transurethral resection of the prostate: what did we learn, and where do we go from here? Eur Urol. 2009; 56: 796-7.

28. Wendt-Nordahl G, Häcker A, Reich O, Djavan B, Alken P, Michel MS: The Vista system: a new bipolar resection device for endourological procedures: comparison with conventional resectoscope. Eur Urol. 2004; 46: 586-90.

29. Ho HS, Yip SK, Lim KB, Fook S, Foo KT, Cheng CW: A prospective randomized study comparing monopolar and bipolar transurethral resection of prostate using transurethral resection in saline (TURIS) system. Eur Urol. 2007; 52: 517-22. 
30. Autorino R, Damiano R, Di Lorenzo G, Quarto G, Perdonà $S$, D'Armiento $\mathrm{M}$, et al.: Four-year outcome of a prospective randomised trial comparing bipolar plasmakinetic and monopolar transurethral resection of the prostate. Eur Urol. 2009; 55: 922-9.

31. Mamoulakis C, Ubbink DT, de la Rosette JJ: Bipolar versus monopolar transurethral resection of the prostate: a systematic review and meta-analysis of randomized controlled trials. Eur Urol. 2009; 56: 798-809.

32. Reich 0, Corvin S, Oberneder R, Sroka R, Muschter $R$, Hofstetter $A$ : In vitro comparison of transurethral vaporization of the prostate (TUVP), resection of the prostate (TURP), and vaporization-resection of the prostate (TUVRP). Urol Res. 2002; 30: 15-20.

33. Gallucci M, Puppo P, Perachino M, Fortunato P, Muto G, Breda $G$, et al.: Transurethral electrovaporization of the prostate vs. transurethral resection. Results of a multicentric, randomized clinical study on 150 patients. Eur Urol. 1998; 33: 359-64.

34. Geavlete B, Georgescu D, Multescu R, Stanescu F, Jecu M, Geavlete P: Bipolar plasma vaporization vs monopolar and bipolar TURP-A prospective, randomized, long-term comparison. Urology. 2011; 78: 930-5.

35. Djavan B, Nickel JC, de la Rosette J, Abrams P: The urologist view of BPH progression: results of an international survey. Eur Urol. 2002; 41: 490-6.

36. Rassweiler J, Schulze M, Stock C, Teber D, De La Rosette $\mathrm{J}$ : Bipolar transurethral resection of the prostate--technical modifications and early clinical experience. Minim Invasive Ther Allied Technol. 2007; 16: 11-21.

37. Reich 0, Gratzke C, Bachmann A, Seitz M, Schlenker B, Hermanek $P$, et al.: Morbidity, mortality and early outcome of transurethral resection of the prostate: a prospective multicenter evaluation of 10,654 patients. J Urol. 2008; 180 : 246-9.

38. Chen Q, Zhang L, Fan QL, Zhou J, Peng YB, Wang Z: Bipolar transurethral resection in saline vs traditional monopolar resection of the prostate: results of a randomized trial with a 2-year follow-up. BJU Int. 2010; 106: 1339-43.

39. Mamoulakis C, Trompetter M, de la Rosette J: Bipolar transurethral resection of the prostate: the 'golden standard' reclaims its leading position. Curr Opin Urol. 2009; 19: 26-32.

40. Chen J, Wang M, Wang S, Sun P, Zhao Y, Zhang L, Jin X: 160Watt lithium triboride laser vaporization versus transurethral resection of prostate: a prospective nonrandomized twocenter trial. Urology. 2012; 79: 650-4.
41. Ruszat R, Seitz M, Wyler SF, Abe C, Rieken M, Reich 0, et al.: GreenLight laser vaporization of the prostate: single-center experience and long-term results after 500 procedures. Eur Urol. 2008; 54: 893-901.

42. Mohanty NK, Vasudeva P, Kumar A, Prakash S, Jain M, Arora RP: Photoselective vaporization of prostate vs. transurethral resection of prostate: A prospective, randomized study with one year follow-up. Indian J Urol. 2012; 28: 307-12.

43. Kaya C, Ilktac A, Gokmen E, Ozturk M, Karaman IM: The longterm results of transurethral vaporization of the prostate using plasmakinetic energy. BJU Int. 2007; 99: 845-8.

44. Botto H, Lebret T, Barré P, Orsoni JL, Hervé JM, Lugagne PM: Electrovaporization of the prostate with the Gyrus device. J Endourol. 2001; 15: 313-6.

45. Dincel C, Samli MM, Guler C, Demirbas M, Karalar M: Plasma kinetic vaporization of the prostate: clinical evaluation of a new technique. J Endourol. 2004; 18: 293-8.

46. Gallucci M, Puppo P, Perachino M, Fortunato P, Muto G, Breda G, et al.: Transurethral electrovaporization of the prostate vs. transurethral resection. Results of a multicentric, randomized clinical study on 150 patients. Eur Urol. 1998; 33: 359-64.

47. Karaman MI, Kaya C, Ozturk M, Gurdal M, Kirecci S, Pirincci N: Comparison of transurethral vaporization using PlasmaKinetic energy and transurethral resection of prostate: 1-year follow-up. J Endourol. 2005; 19: 734-7.

48. Hammadeh MY, Madaan S, Hines J, Philp T: 5-year outcome of a prospective randomized trial to compare transurethral electrovaporization of the prostate and standard transurethral resection. Urology. 200; 61: 1166-71.
Correspondence address: Gholamreza Mokhtari, MD Urology Research Center, School of Medicine, Guilan University of Medical Sciences Urology Research Center, Razi Hospital Sardarjangal Street, Rasht, IR, Iran E mail: gh.mokhtari@yahoo.com 